\title{
1D Bose gases in an optical lattice
}

\section{Journal Article}

\section{Author(s):}

Köhl, M.; Stöferle, T.; Moritz, H.; Schori, S.; Esslinger, T.

Publication date:

2004-12

\section{Permanent link:}

https://doi.org/10.3929/ethz-b-000038171

\section{Rights / license:}

In Copyright - Non-Commercial Use Permitted

Originally published in:

Applied Physics B 79(8), https://doi.org/10.1007/s00340-004-1662-8 

M. KÖHL
T. STÖFERLE
H. MORITZ
C. SCHORI
T. ESSLINGER

\section{D Bose gases in an optical lattice}

\author{
Institute for Quantum Electronics, ETH Zürich Hönggerberg, 8093 Zürich, Switzerland
}

\section{Received: 17 June 2004/Revised version: 7 September 2004 Published online: 20 October 2004 • C Springer-Verlag 2004}

ABSTRACT We report on the study of the momentum distribution of a one-dimensional Bose gas in an optical lattice. From the momentum distribution we extract the condensed fraction of the gas and thereby measure the depletion of the condensate and compare it with a theoretical estimate. We have measured the coherence length of the gas for systems with average occupation $\bar{n}>1$ and $\bar{n}<1$ per lattice site.

PACS 05.30.Jp; 03.75.Kk; 03.75.Lm; 73.43.Nq

1

The one-dimensional Bose gas in an optical lattice

A one-dimensional gas can be created in a trap when the confining potential restricts the motion of the particles to one dimension with the transverse motional degrees of freedom being frozen out. A cigar shaped harmonic trapping geometry is characterized by the frequencies $\omega_{\perp}$ in the two strongly confining axes and $\omega_{\mathrm{ax}}$ in the weakly confining axis. A kinematically one-dimensional situation is achieved when all particles occupy only the ground state in the radial directions, which implies that both the thermal energy $k_{\mathrm{B}} T$ and the interaction energy $\mu$ have to be much smaller than the transverse energy level spacing. In general, one-dimensional systems exhibit increased quantum fluctuations of the phase, such that for a homogeneous 1D gas Bose-Einstein condensation is prevented. For trapped low-dimensional gases the cross-over to a Bose-Einstein condensate takes place at a finite temperature $k_{\mathrm{B}} T_{1 \mathrm{D}}=N \hbar \omega_{\mathrm{ax}} / \ln (2 N)$, where $N$ is the number of particles in the $1 \mathrm{D}$ system $[1,2]$. The fluctuating phase alters the properties of the gas [3-7]. One-dimensional quantum systems exhibit a wealth of fascinating phenomena whose explanations go beyond the mean-field description [8].

One-dimensional trapped Bose-Einstein condensates were recently created and studied [9] using an optical lattice consisting of two mutually perpendicular standing wave laser fields. In this geometry the optical lattice forms an array of one-dimensional tubes, each filled with a Bose-Einstein condensate. This experiment revealed the distinctively different excitation spectrum of a one-dimensional quantum system as compared to its three-dimensional counterpart [10]. In a pre-

Fax: +41-1-6331254, E-mail: esslinger@phys.ethz.ch vious experiment a Bose condensates was loaded into a twodimensional optical lattices to study the coherence between the tubes. In that experiment the tunnel-coupling between adjacent tubes was larger than the axial oscillation frequency, thereby an array of strongly coupled tubes was created [11]. Very recently the lifetime of one-dimensional gases created in an optical lattice were studied [12]. In elongated magnetic and optical traps a regime was accessed where a Bose condensate with $\mu \leq \hbar \omega_{\perp}$ coexisted with a three-dimensional thermal cloud $[13,14]$. In similar elongated traps studies of solitons [15] and of enhanced phase fluctuations have been performed $[16,17]$.

When the one-dimensional Bose gas is exposed to an additional optical lattice potential in axial direction the bosons may become localized in the minima of a periodic potential and the system can then be described by the Bose-Hubbard Hamiltonian $[18,19]$ :

$H=-\widetilde{J} \sum_{\langle i, j\rangle} \hat{a}_{j}^{\dagger} \hat{a}_{i}+\sum_{i} \varepsilon_{i} n_{i}+\frac{U}{2} \sum_{i} n_{i}\left(n_{i}-1\right)$.

$\widetilde{J}$ denotes the hopping matrix element between neighboring lattice sites and determines the rate of which a particle disappears from lattice site $i$ and tunnels to the adjacent lattice site $j\left(\hat{a}_{i}\right.$ and $\hat{a}_{i}^{\dagger}$ are the annihilation and creation operators for an atom at lattice site $i$, respectively). The total tunnel coupling is $J=z \widetilde{J}$ with $z$ being the coordination number of the lattice. The inhomogenity of the atom trap is characterized by $\varepsilon_{i}=\frac{m}{2}\left(i \omega_{\mathrm{ax}} d\right)^{2}$ where $m$ is the atomic mass and $d$ the lattice spacing. The occupation number of lattice site $i$ is denoted $n_{i}$ and $U$ is the onsite-interaction energy between two bosons on the same lattice site. The one-dimensional Bose-Hubbard Hamiltonian exhibits a transition from a superfluid phase to a Mott insulating phase for a ratio $(U / J)_{\mathrm{c}} \simeq 2[3-6]$, which we experimentally demonstrated recently [7]. Due to stronger quantum fluctuations of the phase in a one-dimensional quantum system this value is lower than the corresponding critical value in three dimensions $(U / J)_{\mathrm{c}}=5.8[18-20]$. Tuning the depth of the periodic potential changes the parameter $U / J$, which leads to increased effects of interparticle interactions and small filling of the lattice sites may turn the system into a gas of hardcore bosons $[21,22]$. In the inhomogeneous system Mott insulating regions with commensurate filling coexist with superfluid regions with incommensurate filling and the insulator is formed in a crossover type transition [4]. 
2

2.1

\section{Experimental setup}

\section{Bose condensates in an optical lattice}

In the experiment, we collect up to $2 \times 10^{9}{ }^{87} \mathrm{Rb}$ atoms in a vapor cell magneto-optical trap. After polarization gradient cooling and optical pumping into the $\left|F=2, m_{F}=2\right\rangle$ hyperfine ground state the atoms are captured in a magnetic quadrupole trap. After magnetic transport of the trapped atoms over a distance of $40 \mathrm{~cm}$ the magnetic trapping potential is converted into the harmonic and elongated potential of a QUIC trap [23]. Subsequently, we perform radio frequency induced evaporation of the cloud over a period of $25 \mathrm{~s}$. After evaporation we observe almost pure three-dimensional BoseEinstein condensates of up to $1.5(0.2) \times 10^{5}$ atoms. Following the condensation we adiabatically change the trapping geometry to an approximately spherical symmetry with trapping frequencies of $\omega_{x}=2 \pi \times 18 \mathrm{~Hz}, \omega_{y}=2 \pi \times 20 \mathrm{~Hz}$, and $\omega_{z}=2 \pi \times 22 \mathrm{~Hz}$. This reduces the peak density by a factor of four and allows us to load the optical lattice more uniformly.

The optical lattice is formed by three retro-reflected laser beams. Each beam is derived from a laser diode at a wavelength of $\lambda=826 \mathrm{~nm}$. At the position of the condensate the beams are circularly focused to $1 / \mathrm{e}^{2}$-radii of $120 \mu \mathrm{m}(x$ and $y$ axes) and $105 \mu \mathrm{m}(z)$. The three beams possess mutually orthogonal polarizations and their frequencies are offset with respect to each other by several ten MHz. We stabilize the lasers to a high-finesse Fabry-Pérot cavity, thereby reducing their line width to $\sim 10 \mathrm{kHz}$. In order to load the condensate into the ground state of the optical lattice, the intensities of the lasers are slowly increased to their final values using an exponential ramp with a time constant of $25 \mathrm{~ms}$ and a duration of $100 \mathrm{~ms}$. The resulting optical potential depths $V_{x, y, z}$ are proportional to the laser intensities and are conveniently expressed in terms of the recoil energy $E_{\mathrm{R}}=\frac{\hbar^{2} k^{2}}{2 m}$ with $k=\frac{2 \pi}{\lambda}$.

\section{2}

\section{Preparation of one-dimensional quantum gases}

Using the optical lattice we realize one-dimensional quantum gases ordered in an array of one-dimensional tubes. Two lattice axes are ramped to a fixed value $V_{\perp} \equiv V_{x}=V_{z}=$ $30 E_{\mathrm{R}}$. In this configuration the transverse tunnelling rates $J_{x}$ and $J_{z}$ are small compared to the duration of the experiment. We therefore neglect tunnelling between neighboring tubes and assume an array of isolated 1D gases. An additional optical lattice is applied along the symmetry axis with a potential depth $V_{\mathrm{ax}} \equiv V_{y} \ll V_{\perp}$. The potential depth $V_{\mathrm{ax}}$ controls the value of $U / J$.

\section{$3 \quad$ Results \\ 3.1 \\ Momentum distribution}

We have studied the momentum distribution of the gas by imaging the expanding atom cloud after being released from the optical lattice. Prior to switching off the lattice the laser intensity of the axial lattice is increased to $\simeq 25 E_{\mathrm{R}}$ for $40 \mu \mathrm{s}$. Then all laser beams are extinguished simultaneously within $5 \mu$ s and the magnetic trapping potential is switched off within $300 \mu \mathrm{s}$. This procedure may be regarded as a projection of the wave function of the atoms onto the Bloch momentum states for a lattice depth of $25 E_{\mathrm{R}}$. This method offers the advantage of controlling adiabatic processes during the switch-off of the lattice. These might affect the momentum distribution of the released atoms [22].

For our one-dimensional gases the collisional interaction during expansion - which would give rise to a Thomas-Fermi type envelope of the density distribution in the $y$-direction is small. This is related to the extremely fast radial expansion of the clouds, which are initially confined to the harmonic oscillator ground state $a_{\perp}=\sqrt{\hbar /\left(m \omega_{\perp}\right)}$ with a trapping frequency of $\omega_{\perp}=2 \pi \times 33 \mathrm{kHz}$. The peak density decreases proportional to $1 / \sqrt{1+\left(\omega_{\perp} t\right)^{2}}$ which is much faster than the timescale for axial expansion that is given by the axial trapping frequency of $\omega_{\mathrm{ax}}=2 \pi \times 80 \mathrm{~Hz}$. This axial confinement is introduced by the gaussian waist of the laser beams forming the array of one-dimensional tubes.

We have studied the resulting momentum distribution after $15 \mathrm{~ms}$ of free expansion of the cloud. The atoms were imaged by resonant absorption imaging and we determine the optical density in the center of the cloud by averaging over a $62 \mu \mathrm{m}$ wide section in the image. Figure 1 shows the measured momentum distribution for a sample with an average filling of $\bar{n}=1.2$ atoms per lattice site. We calculate the average filling from the mean density of atoms which is derived known atom number and the measured trapping frequencies and includes a modified coupling strength due to the localization of the atoms in the optical lattice potential [26]. The atom number distribution at a given lattice site in the superfluid phase can be approximated by a Poisson distribution. Therefore, even with an average occupation number of $\bar{n}=0.6$ the probability of finding doubly or multiply occupied lattice sites is on the order of $10 \%$.

\subsection{Quantum depletion of the condensate}

When the potential depth of the lattice is raised the strength of the atom-atom interaction increases. This is accompanied by quantum depletion of the condensate [24]. The

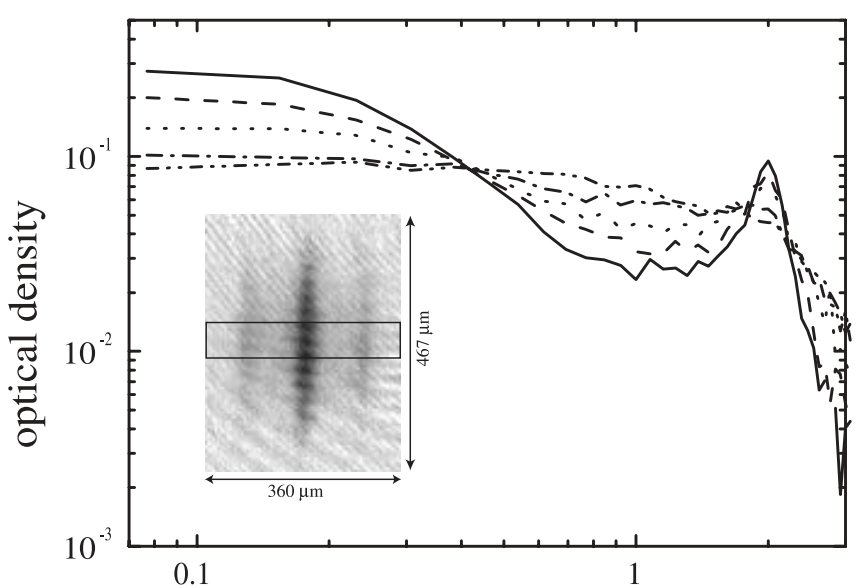

momentum [hk]

FIGURE 1 Measured momentum distribution for $N=1.5 \times 10^{5}$ atoms for different depths of the axial lattice potential. Top to bottom on the left hand site: $V_{\mathrm{ax}}=5, V_{\mathrm{ax}}=7, V_{\mathrm{ax}}=9, V_{\mathrm{ax}}=12, V_{\mathrm{ax}}=18$ (in units of $E_{\mathrm{R}}$ ). The inset shows an absorption image after $10 \mathrm{~ms}$ time of flight where the area of averaging is indicated 


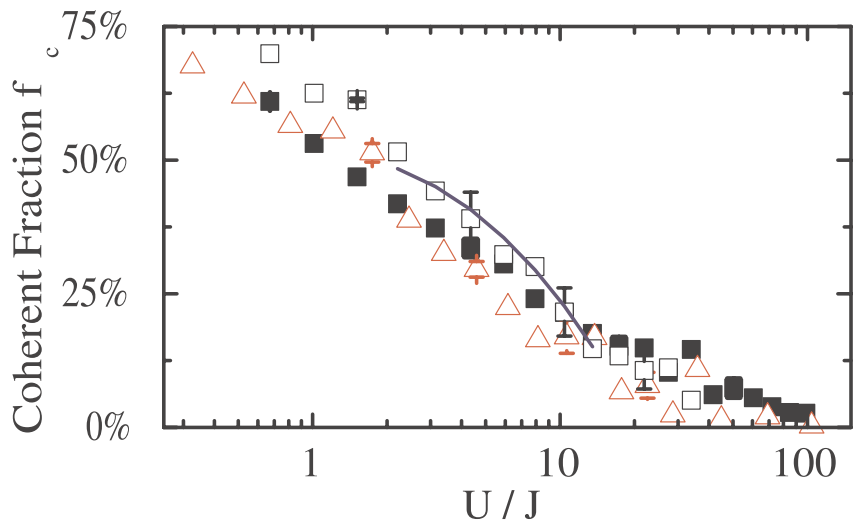

FIGURE 2 Coherent fraction of the 1D Bose gas as measured from the time-of-flight image. One-dimensional system with $\bar{n}=1.2$ (solid squares), one-dimensional system with $\bar{n}=0.6$ (open squares), three-dimensional gas with $\bar{n}=1$ (triangles). Part of the experimental data are taken from [7]. The solid line shows the calculated quantum depletion according to (2) using $N_{\mathrm{W}}=40$

reduced condensate fraction of the system diminishes the contrast in the matter wave interference pattern after the atoms have been released from the optical lattice. To extract the number of coherent atoms $N_{\text {coh }}$ from the interference pattern, the interference peaks at $0 \hbar k, \pm 2 \hbar k$ and $\pm 4 \hbar k$ are fitted by gaussians. Incoherent atoms, both due to localization of the atoms in the lattice or due to depletion of the condensate, give rise to a broad gaussian background centered at zero quasi momentum which dominates for higher $V_{\mathrm{ax}}$ [25]. Taking this fit as a measure of the number of incoherent atoms $N_{\text {incoh }}$, we calculate the coherent fraction $f_{c}=\frac{N_{\text {coh }}}{N_{\text {coh }}+N_{\text {incoh }}}$ [7]. The measured data are shown in Fig. 2. For the one-dimensional Bose gas in an optical lattice the quantum depletion is calculated by [26]

$\frac{n}{n_{0}}=\sqrt{\frac{1}{2 \pi^{2}} \frac{m}{m^{*}} \frac{1}{a_{1 \mathrm{D}} n_{1 \mathrm{D}}}} \ln \left(\frac{4 N_{\mathrm{w}}}{\pi}\right)$.

Here $m$ is the atomic mass, $m^{*}$ is the effective mass in the lattice, $n_{1 \mathrm{D}}$ is the $1 \mathrm{D}$ density, $a_{1 \mathrm{D}}$ is the one-dimensional scattering length [27] and $N_{\mathrm{w}}$ is the number of potential wells occupied by atoms. This expression holds in the tight binding regime but for small depletion. Figure 2 displays the measured coherent fraction together with the calculated values for the quantum depletion, which show good agreement in the range of validity of (2).

\subsection{Coherence length}

From the width of the central coherent momentum component the coherence length of the gas in the optical lattice can be inferred. A Bose-Einstein condensate exhibits a long coherence length which leads to a small width of the interference peak. Due to the inhomogeneity of the trap the transition to the Mott insulator sets in at those positions, where the local density becomes commensurable with the spacing of the potential wells in the optical lattice. This process breaks up the condensed cloud into smaller units and therefore reduces the coherence length of the system. In Fig. 3 we show the change of the coherence length as a function of the parameter $U / J$. For the one-dimensional gas with an average occupancy of

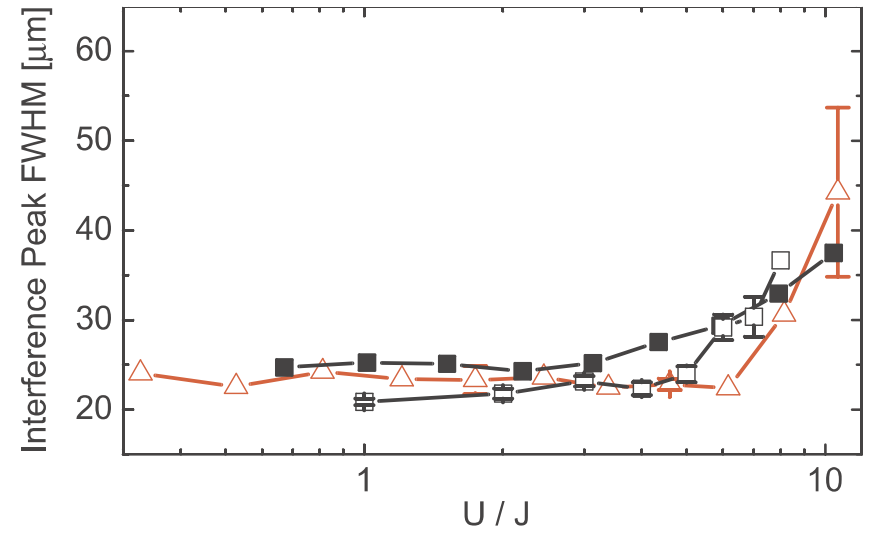

FIGURE 3 Width of the central momentum peak as a measure of the phase coherence length of the system. One-dimensional system with $\bar{n}=1.2$ (solid squares), one-dimensional system with $\bar{n}=0.6$ (open squares), threedimensional gas with $\bar{n}=1$ (triangles). Part of the data is taken from [7]

$\bar{n}=1.2$ we observe a kink in the coherence length at $U / J \simeq 2$ which indicates the onset of the Mott insulating phase. For the three-dimensional gas with $\bar{n}=1$ we find the Mott insulator transition at $U / J \simeq 6$, in agreement with the mean field theory. For very small one-dimensional systems with $\bar{n}=0.6$ we observe that the coherence length of the sample starts to increase around $U / J=4$. This is in qualitative agreement with the theoretical prediction that the Mott-insulator transition occurs for $U / J \simeq 2$ only for a chemical potential corresponding a density $\bar{n}=1$ but at larger values for $\bar{n}<1$ [3].

\section{$4 \quad$ Conclusion}

We have studied the momentum distribution of one-dimensional Bose gases in an optical lattice for different average occupation of the lattice sites. We find that the coherent fraction of atoms is independent of the atomic density when we tune the interaction strength $U / J$ from the superfluid to the Mott-insulating regime. The change of the coherence length appear to indicate that the system enters the Mottinsulator for small atomic densities only for larger values of $U / J$.

ACKNOWLEDGEMENTS We acknowledge stimulating discussions with Thierry Giamarchi, Corinna Kollath and Stefan Wessel and support from SNF and QSIT.

\section{REFERENCES}

1 W. Ketterle, N.J. van Druten: Phys. Rev.A 54, 61 (1996)

2 D.S. Petrov, G.V. Shlyapnikov, J.T.M. Walraven: Phys. Rev. Lett. 85, 3745 (2000)

3 T.D. Kühner, H. Monien: Phys. Rev. B 58, 14741 (1998)

4 G.G. Batrouni, V. Rousseau, R.T. Scalettar, M. Rigol, A. Muramatsu, P.J.H. Denteneer, M. Troyer: Phys. Rev. Lett. 89, 117203 (2002)

5 C. Kollath, U. Schollwöck, J. von Delft, W. Zwerger: Phys. Rev. A 69, 031601 (2004)

6 S. Wessel, F. Alet, M. Troyer, G.G. Batrouni: e-print cond-mat/0404552 (2004)

7 T. Stöferle, H. Moritz, C. Schori, M. Köhl, T. Esslinger: Phys. Rev. Lett. 92, 130403 (2004)

8 For a review see: T. Giamarchi: Quantum Physics in One Dimension (Oxford 2004)

9 H. Moritz, T. Stöferle, M. Köhl, T. Esslinger: Phys. Rev. Lett. 91, 250402 (2003)

10 C. Menotti, S. Stringari: Phys. Rev. A. 66, 043610 (2002) 
11 M. Greiner, I. Bloch, O. Mandel, T.W. Hänsch, T. Esslinger: Phys. Rev. Lett. 87, 160405 (2001)

12 B. Laburthe-Tolra, K.M. O’Hara, J.H. Huckans, W.D. Phillips, S.L. Rolston, J.V. Porto: Phys. Rev. Lett. 92, 190401 (2004)

13 A. Görlitz, J.M. Vogels, A.E. Leanhardt, C. Raman, T.L. Gustavson, J.R. Abo-Shaeer, A.P. Chikkatur, S. Gupta, S. Inouye, T. Rosenband, W. Ketterle: Phys. Rev. Lett. 87, 130402 (2001)

14 F. Schreck, L. Khaykovich, K.L. Corwin, G. Ferrari, T. Bourdel, J. Cubizolles, C. Salomon: Phys. Rev. Lett. 87, 080403 (2001)

15 K.E. Strecker, G.B. Partridge, A.G. Truscott, R.G. Hulet: Nature 417, 150 (2002)

16 S. Dettmer, D. Hellweg, P. Ryytty, J.J. Arlt, W. Ertmer, K. Sengstock, D.S. Petrov, G.V. Shlyapnikov, H. Kreutzmann, L. Santos, M. Lewenstein: Phys. Rev. Lett. 87160406 (2001)

17 S. Richard, F. Gerbier, J.H. Thywissen, M. Hugbart, P. Bouyer, A. Aspect: Phys. Rev. Lett. 91, 010405 (2003)
18 M.P.A. Fischer, P.B. Weichmann, G. Grinstein, D.S. Fisher: Phys. Rev. B 40, 546 (1989)

19 D. Jaksch, C. Bruder, J.I. Cirac, C.W. Gardiner, P. Zoller: Phys. Rev. Lett. 81, 3108 (1998)

20 M. Greiner, O. Mandel, T. Esslinger, T.W. Hänsch, I. Bloch: Nature 415, 39 (2002)

21 M.A. Cazalilla: Phys. Rev. A 67, 053606 (2003)

22 B. Paredes, A. Widera, V. Murg, O. Mandel, S. Fölling, I. Cirac, G.V. Shlyapnikov, T.W. Hänsch, I. Bloch: Nature 429, 277 (2004)

23 T. Esslinger, I. Bloch, T.W. Hänsch: Phys. Rev. A 58, 2664 (1998)

24 N. Bogoliubov: J. Phys. USSR 11, 23 (1947)

25 Z. Hadzibabic, S. Stock, B. Battelier, V. Bretin, J. Dalibard: quant$\mathrm{ph} / 0405113$ (2004)

26 M. Krämer, C. Menotti, L. Pitaevskii, S. Stringari: Eur. Phys. J. D 27, 247 (2003)

27 M. Olshanii: Phys. Rev. Lett. 81, 938 (1998) 